\title{
Engagement no Trabalho em Residentes Médicos de Pediatria
}

\section{Work Engagement Among Pediatric Physician Residents}

\author{
Priscila Regina Teixeira ${ }^{I}$ \\ Luciano Garcia Lourenção ${ }^{I}$ \\ Cláudia Eli Gazetta ${ }^{I}$ \\ Elizangela Gianini Gonsalez \\ Daniela Salvagni Rotta \\ Maria Helena Pinto ${ }^{I}$ \\ Lilian Peres ${ }^{I}$ \\ Denise Beretta
}

\section{PALAVRAS-CHAVE}

- Médicos Residentes.

- Internato e Residência.

- Educação Médica.

- Satisfação no Trabalho.

Recebido em: 02/07/2016

Aprovado em: 01/11/2016
REVISTA BRASLLEIRA DE EDUCAÇÃO MÉDICA

\section{RESUMO}

Objetivos: Avaliar os indices de engagement no trabalho em residentes médicos de Pediatria. Métodos: Amostra composta por 36 profissionais matriculados no programa de residência médica em Pediatria da Faculdade de Medicina de São José do Rio Preto no ano de 2013. Os dados foram coletados de setembro de 2013 a fevereiro de 2014, utilizando-se a Utrecht Work Engagement Scale (Uwes) - Escala Utrecht de Engajamento no Trabalho -, composta por 17 itens de autoavaliação com três dimensões, Vigor, Dedicação e Absorção, além de um escore geral do constructo. Resultados: 91,67\% eram do sexo feminino; a idade mediana foi de 28 anos (mínimo: 25; máximo: 34); 86,11\% eram solteiros; 33,33\% tinham renda familiar de 2 a 5 salários, e 44,44\%, mais de 10 salários mínimos; 88,89\% estavam satisfeitos com o trabalho; e 52,78\% já pensaram em desistir do programa. Os indices de engagement variaram de 3,56 a 4,28. A dimensão Dedicação obteve índice alto $(4,28 \pm 1,12)$, $e$ as dimensões Absorção, Vigor e Engagement, médios (3,58 1,$00 ; 3,56 \pm 0,98 ; 3,78 \pm 0,96)$. Quanto ao desejo de desistir do programa, ambos os grupos apresentaram índices médios para as dimensões Absorção e Vigor; nas dimensões Dedicação e Engagement, indices médios para os que já pensaram em desistir do programa e altos para aqueles que nunca pensaram em desistir. Sobre a satisfação com o programa, na dimensão Dedicação, os indices foram: alto para os satisfeitos e médio para os não satisfeitos (4,49 e 2,6); as dimensões Absorção, Vigor e Engagement apresentaram indices médios para ambos os grupos - 3,66, 3,68 e 3,92 entre os satisfeitos e 2,95, 2,54 e 2,7 para os não satisfeitos, respectivamente. Conclusão: Os residentes em Pediatria da Faculdade de Medicina de São José do Rio Preto apresentaram bons indices de engagement, principalmente no domínio Dedicação. Há um baixo percentual de profissionais com baixo indice de engagement em todos os domínios da Uwes. 


\section{KEYWORDS}

- Medical Staff, Hospital.

- Internship and Residency.

- Medical Education.

- Job Satisfaction.

\section{ABSTRACT}

Objectives: To evaluate work engagement rates among medical residents in pediatrics. Methods: Data was collected from a sample of 36 professionals enrolled in residency programs in pediatrics at the Medical School of São José do Rio Preto from September 2013 to February 2014, using the Utrecht Work Engagement Scale (Uwes), featuring seventeen items for self assessment and three dimensions: vigor, dedication, and absorption, and an overall score for the construct. Results: $91.67 \%$ were female, the median age was 28 years (minimum: 25, maximum: 34), 86.11\% single, 33.33\% had a family income of 2-5 monthly wages and $44.44 \%$ over 10 minimum wages, $88.89 \%$ were satisfied with their work and $52.78 \%$ were considering quitting the program. The engagement of the indexes ranged from 3.56 to 4.28. Dedication was high (4.28 \pm 1.12$)$, while Absorption, Vigor, and Engagement, were medium $(3.58 \pm 1.00,3.56 \pm 0.98$ and $3.78 \pm 0.96)$. Regarding the desire to withdraw from the program, both groups revealed average rates for Absorption and Vigor; while Dedication and Engagement rates were average among those to have considered quitting the program, and high among those who had never thought about quitting. In terms of satisfaction with the program, Dedication indexes were high for those happy on the residency and medium for those not satisfied (4.49 and 2.6). Absorption, Vigor, and Engagement rates were average for both groups - 3.66, 3.68, and 3.92 among satisfied residents and 2.95, 2.54, and 2.7 among those not satisfied, respectively. Conclusion: Pediatrics residents at the Medical School of São José do Rio Preto revealed sound levels of engagement, particularly in terms of Dedication. A low percentage of professionals was revealed to have low engagement rates across all areas of the Uwes.

\section{INTRODUÇÃO}

A necessidade de adquirir habilidades e mais conhecimentos que garantissem maior segurança ao exercício da profissão médica originou a residência médica, uma modalidade de ensino caracterizada por treinamento em serviço sob supervisão, em tempo integral, considerada a melhor maneira de aperfeiçoamento e especialização na área da saúde ${ }^{1,2}$. O primeiro programa foi criado no Hospital John Hopkins, nos Estados Unidos, em Cirurgia e Clínica Médica ${ }^{1,3}$.

No Brasil, a residência médica iniciou-se na década de 1940, com a criação dos primeiros programas para suprir a necessidade de os profissionais aprimorarem suas competências, atendendo à demanda do mercado de trabalho, que se tornava competitivo. No final da década de 1960 e na década de 1970, o aumento do número de cursos de Medicina no País ocasionou a expansão dos programas de residência médica, que passaram a ser amplamente procurados por profissionais egressos das escolas médicas ${ }^{3}$.

$O$ perfil e a tendência de formação dos médicos sofrem grande influência das práticas sanitárias, da organização do sistema de saúde e do mercado de trabalho, o que torna primordial a compreensão do papel que atualmente a residência médica tem na formação desses profissionais ${ }^{3,4}$.

A literatura especializada sobre o tema mostra que os profissionais que se inserem nos programas de residência passam por grandes dificuldades para concluírem o processo de for- mação. Estudos sobre saúde e qualidade de vida de médicos residentes apontam que, embora proporcionem grande desenvolvimento profissional e pessoal, os programas de residência médica compreendem um processo desgastante, com altos níveis de estresse profissional, longas jornadas de trabalho e organização inadequada, causando problemas de saúde que interferem na qualidade de vida desses profissionais ${ }^{2,5-6}$.

Embora muitos estudos focalizem problemas, dificuldades e comprometimento da saúde dos residentes médicos, muitas vezes mostrando resultados negativos para os profissionais, a residência médica é considerada o melhor método de capacitação profissional ${ }^{2-6}$.

A experiência com a residência médica tem evidenciado que, embora o treinamento possa causar efeitos prejudiciais na sensibilidade dos médicos em relação aos pacientes, no desempenho acadêmico e profissional, na saúde, no bem-estar e na qualidade de vida pessoal, há contribuições, como o incremento de competências, autoconfiança e segurança profissionais ${ }^{2,5-6}$.

Para Rodríguez-Montalbánet al. ${ }^{7}$, a avaliação e a prevenção de riscos psicossociais prevalentes nas organizações, como estresse, burnout e assédio, são importantes para o desenvolvimento institucional. Contudo, os autores destacam a relevância de analisar e melhorar os pontos fortes, como satisfação, envolvimento com o trabalho e emoções positivas. Assim, estudar os aspectos positivos do ser humano se torna tão importante quanto investigar os aspectos negativos ${ }^{8}$. 
Destacam-se como consequências positivas da relação do profissional com o ambiente de trabalho: aumento da produtividade, maior disposição, interação com os colegas, motivação e criatividade, redução do absenteísmo e diminuição de $\operatorname{erros}^{8-11}$.

Considerando, portanto, a escassez de pesquisas sobre engagement no trabalho e os aspectos inerentes à atuação médica, este estudo se torna relevante. Avaliar os índices de engagement no trabalho em profissionais matriculados no programa de residência médica em Pediatria permitirá conhecer a relação desses profissionais com o trabalho, subsidiando ações que fortaleçam o processo de formação e, consequentemente, a qualidade do atendimento prestado aos pacientes.

Este estudo objetivou avaliar os índices de engagement no trabalho dos profissionais matriculados no programa de residência médica em Pediatria da Faculdade de Medicina de São José do Rio Preto (SP).

\section{METODOLOGIA}

Trata-se de um estudo transversal, de realizado com 36 profissionais matriculados no programa de residência médica em Pediatria da Faculdade de Medicina de São José do Rio Preto, no ano de 2013, que consentiram em participar da pesquisa depois de informados sobre seus objetivos e finalidade.

Os dados foram coletados de setembro de 2013 a fevereiro de 2014, utilizando-se dois instrumentos: um elaborado pelos autores, contendo dados pessoais - idade, sexo, peso, altura, estado civil, escolaridade, renda familiar, se está satisfeito e se já pensou em desistir do programa - para elaboração do perfil dos profissionais; e a Utrecht Work Engagement Scale (Uwes), composta por 17 itens de autoavaliação com três dimensões - Vigor, Dedicação e Absorção -, além de um escore geral do constructo ${ }^{12}$.

O Vigor se refere a altos níveis de energia e resiliência, à vontade de investir esforços, não se fadigar com facilidade e persistir em face das dificuldades. Aqueles que apresentam altos escores nesta dimensão geralmente possuem muita energia quando trabalham, enquanto aqueles que apresentam baixos escores têm menos energia ${ }^{9-12}$.

A Dedicação está relacionada a um senso de significado pelo trabalho, a sentir-se entusiasmado e orgulhoso em relação ao seu labor, sentindo-se inspirado e desafiado por ele. Profissionais com altos escores nesta dimensão identificam-se fortemente com o seu trabalho porque a experiência é significativa, inspiradora e desafiadora. Além disso, geralmente sentem-se entusiasmados e orgulhosos em relação ao seu trabalho. Aqueles que apresentam baixos escores não se identificam com o seu trabalho porque não o experienciam como significativo, inspirador ou desafiador, e não se sentem nem entusiasmados nem orgulhosos em relação a $\operatorname{ele}^{9-12}$.
A Absorção se refere a estar totalmente imerso em seu trabalho e com dificuldades em desapegar-se dele; o tempo passa rapidamente, e o profissional se esquece de tudo ao seu redor. Apresentar índices de Absorção elevados implica sentir-se envolvido e imerso no trabalho, com dificuldades em desapegar-se dele. Consequentemente, tudo ao redor é esquecido, e o tempo parece voar ${ }^{9-12}$.

Os dados sociodemográficos foram utilizados para caracterizar a população do estudo. Para análise do engagement no trabalho, os cálculos dos escores foram realizados conforme o modelo estatístico proposto no Manual Preliminar Uwes - Utrecht Work Engagement Scale ${ }^{12}$, apresentando-se valores mínimo, máximo, mediana, média ( \pm desvio padrão) e intervalo de confiança de 95\% para cada dimensão da Uwes. Realizou-se, então, a interpretação dos valores obtidos, a partir decodificação do Manual Preliminar Uwes, como apresentado no Quadro 1.

\begin{tabular}{|c|c|c|}
\hline \multicolumn{3}{|c|}{$\begin{array}{c}\text { QUADRo } 1 \\
\text { Modelo para interpretação dos valores } \\
\text { dos escores médios do estudo }\end{array}$} \\
\hline Classificação & \multicolumn{2}{|c|}{$\begin{array}{c}\text { Frequência dos sentimentos em relação ao } \\
\text { trabalho (questões Uwes) }\end{array}$} \\
\hline Muito baixo & 0 a 0,99 & $\begin{array}{l}=0 \text { (nenhuma vez) } \\
=1 \text { (algumas vezes por ano) }\end{array}$ \\
\hline Baixo & 1 a 1,99 & $=2$ (uma vez ou menos por mês) \\
\hline \multirow{2}{*}{ Médio } & 2 a 2,99 & $=3$ (algumas vezes por mês) \\
\hline & 3 a 3,99 & $=4$ (uma vez por semana) \\
\hline Alto & 4 a 4,99 & $=5$ (algumas vezes por semana) \\
\hline Muito alto & 5 a 6 & $=6$ (todos os dias $)$ \\
\hline
\end{tabular}

A análise dos dados foi realizada com o programa Statistical Package for Social Sciences (SPSS), versão 17.0. As comparações foram feitas pelo teste qui-quadrado, considerando significante um valor-p igual ou inferior a 0,05.

Respeitando os preceitos Éticos de Pesquisas envolvendo seres humanos, este projeto foi submetido à apreciação do Comitê de Ética em Pesquisa da Faculdade de Medicina de São José do Rio Preto, sob o Protocolo CAAE 23948113.2.0000.5415 e aprovado em 12 de novembro de 2013 com o Parecer nํㅜㄴ. 460.331 .

\section{RESULTADOS}

Participaram do estudo 36 médicos residentes em Pediatria. Observou-se prevalência do sexo feminino (33 - 91,67\%). A faixa etária variou de 25 a 34 anos, sendo a idade mediana de 28 anos. Em relação ao estado civil, observou-se que 31 $(86,11 \%)$ profissionais eram solteiros. Quanto à renda familiar, $12(33,33 \%)$ tinham renda familiar de 6 a 10 salários mínimos, e 16 (44,44\%), acima de 10 salários mínimos (Tabela 1). 
TABELA 1

Distribuição das características sociodemográficas dos médicos matriculados no programa de residência médica em Pediatria da Faculdade de Medicina de São José do Rio Preto. São José do Rio Preto, 2013-2014

\begin{tabular}{|c|c|c|c|}
\hline & & $\mathbf{n}$ & $\%$ \\
\hline \multirow{2}{*}{ Sexo } & Masculino & 3 & 8,33 \\
\hline & Feminino & 33 & 91,67 \\
\hline \multirow{4}{*}{ Faixa etária } & $20-25$ anos & 1 & 2,78 \\
\hline & $26-30$ anos & 26 & 72,22 \\
\hline & $31-35$ anos & 5 & 13,89 \\
\hline & Não respondeu & 4 & 11,11 \\
\hline \multirow{2}{*}{ Estado civil } & Casado & 5 & 13,89 \\
\hline & Solteiro & 31 & 86,11 \\
\hline \multirow{4}{*}{$\begin{array}{l}\text { Renda familiar } \\
\text { (salários mínimos) }\end{array}$} & 2 a 5 & 6 & 16,67 \\
\hline & 6 a 10 & 12 & 33,33 \\
\hline & Mais de 10 & 16 & 44,44 \\
\hline & Não respondeu & 2 & 5,56 \\
\hline \multirow{2}{*}{$\begin{array}{l}\text { Satisfeito com o } \\
\text { programa }\end{array}$} & Sim & 32 & 88,89 \\
\hline & Não & 4 & 11,11 \\
\hline \multirow{2}{*}{$\begin{array}{l}\text { Pensou em desistir } \\
\text { do programa }\end{array}$} & Sim & 19 & 52,78 \\
\hline & Não & 17 & 47,22 \\
\hline
\end{tabular}

Destaca-se, ainda, que $32(88,89 \%)$ profissionais se disseram satisfeitos com o programa em que estavam matriculados, embora 19 (52,78\%) já tenham pensado em desistir do programa.

A Tabela 2 apresenta os resultados da avaliação dos índices de engagement no trabalho com base na distribuição dos valores mínimo, máximo, mediana, média ( \pm desvio padrão) e intervalo de confiança de $95 \%$, segundo as dimensões da Uwes: Vigor, Dedicação, Absorção e Engagement. A média das dimensões variou de 3,56 a 4,28, e os desvios padrão variaram entre 0,96 e 1,12. A dimensão Dedicação obteve a maior média $(4,28 \pm 1,12)$, classificada como alta. As dimensões Absorção, Vigor e Engagement obtiveram valores classificados como médios $-3,58 \pm 1,00 ; 3,56 \pm 0,98 ; 3,78 \pm 0,96$, respectivamente.

$\mathrm{Na}$ análise da confiabilidade, os valores do Coeficiente Alfa de Cronbach variaram de 0,711 a 0,916, conforme mostra a Tabela 3. Essa tabela também apresenta a distribuição do número de profissionais em cada nível de classificação do engagement no trabalho. Destaca-se que, na dimensão Dedicação, 69,45\% ( $\mathrm{N}=25)$ dos profissionais apresentaram nível de engagement alto ou muito alto. As dimensões Vigor e Absorção apresentaram, cada uma, 58,33\% ( $\mathrm{N}=21)$ dos profissionais com nível de engagement médio. Para a dimensão Engagement, o ní-

\section{TABELA 2}

Distribuição dos valores mínimo, máximo, mediana, média ( \pm desvio padrão) e intervalo de confiança de $95 \%$ para as dimensões da Utrecht Work Engagement Scale (Uwes), segundo a avaliação dos profissionais matriculados no programa de residência médica em Pediatria da Faculdade de Medicina de São José do Rio Preto. São José do Rio Preto, 2013-2014

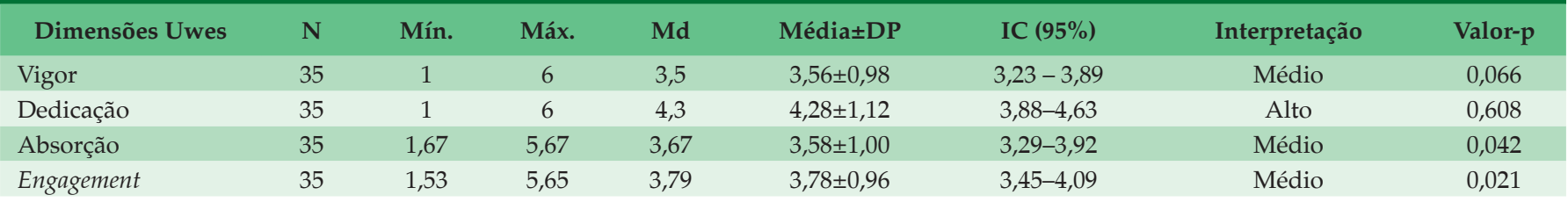

Legenda: Mín.: mínimo, Máx.: máximo, Md: mediana, DP: desvio padrão, IC 95\%: intervalo de confiança de 95\%.

\begin{tabular}{|c|c|c|c|c|c|c|c|c|c|c|}
\hline \multicolumn{11}{|c|}{$\begin{array}{l}\text { Distribuição do coeficiente de confiabilidade, escore médio e número de profissionais, segundo classificação do nível } \\
\text { de engagement para as dimensões da Utrecht Work Engagement Scale (Uwes). São José do Rio Preto, 2013-2014 }\end{array}$} \\
\hline \multirow{3}{*}{ Dimensões Uwes } & \multirow{3}{*}{\multicolumn{2}{|c|}{ Alfa de Cronbach Escore Médio }} & \multicolumn{8}{|c|}{ Classificação do Nível de Engagement no Trabalho } \\
\hline & & & \multicolumn{2}{|c|}{ Baixo } & \multicolumn{2}{|c|}{ Médio } & \multicolumn{2}{|c|}{ Alto } & \multicolumn{2}{|c|}{ Muito Alto } \\
\hline & & & $\mathbf{N}$ & $\%$ & $\mathbf{N}$ & $\%$ & $\mathbf{N}$ & $\%$ & $\mathbf{N}$ & $\%$ \\
\hline Vigor & 0,747 & 3,56 & 2 & 5,55 & 21 & 58,33 & 10 & 27,78 & 3 & 8,33 \\
\hline Dedicação & 0,898 & 4,28 & 2 & 5,55 & 9 & 25 & 15 & 41,67 & 10 & 27,78 \\
\hline Absorção & 0,711 & 3,58 & 2 & 5,55 & 21 & 58,33 & 8 & 22,22 & 5 & 13,89 \\
\hline Engagement & 0,916 & 3,78 & 2 & 5,55 & 17 & 47,22 & 13 & 36,11 & 4 & 11,11 \\
\hline
\end{tabular}


TABELA 4

Distribuição das médias ( \pm desvio padrão) para as escalas da Utrecht Work Engagement Scale (Uwes), segundo desejo de desistir e satisfação referida pelos profissionais matriculados no programa de residência médica em Pediatria da Faculdade de Medicina de São José do Rio Preto. São José do Rio Preto, 2013-2014

\begin{tabular}{|c|c|c|c|c|c|}
\hline Dimensão da Uwes & Pensou em Desistir & $\mathbf{N}$ & Média $\pm D P$ & Interpretação & $\begin{array}{l}\text { Valor-p } \\
\text { (t-test) }\end{array}$ \\
\hline \multirow{2}{*}{ Vigor } & Sim & 19 & $3,23 \pm 0,82$ & Médio & 0,034 \\
\hline & Não & 17 & $3,92 \pm 1,03$ & Médio & \\
\hline \multirow{2}{*}{ Dedicação } & Sim & 19 & $3,92 \pm 1,13$ & Médio & 0,038 \\
\hline & Não & 16 & $4,7 \pm 0,98$ & Alto & \\
\hline \multirow{2}{*}{ Absorção } & Sim & 19 & $3,36 \pm, 097$ & Médio & 0,178 \\
\hline & Não & 17 & $3,82 \pm 1,01$ & Médio & \\
\hline \multirow{2}{*}{ Engagement } & Sim & 19 & $3,48 \pm 0,88$ & Médio & 0,044 \\
\hline & Não & 16 & $4,14 \pm 0,96$ & Alto & \\
\hline Dimensão da Uwes & Satisfação com o Programa & $\mathrm{N}$ & Média_DP & Interpretação & $\begin{array}{l}\text { Valor-p } \\
\text { (t-test) }\end{array}$ \\
\hline \multirow{2}{*}{ Vigor } & Sim & 32 & $3,68 \pm 0,87$ & Médio & 0,025 \\
\hline & Não & 4 & $2,54 \pm 1,30$ & Médio & \\
\hline \multirow{2}{*}{ Dedicação } & Sim & 31 & $4,49 \pm 0,91$ & Alto & 0,001 \\
\hline & Não & 4 & $2,6 \pm 1,29$ & Médio & \\
\hline \multirow{2}{*}{ Absorção } & Sim & 32 & $3,66 \pm 0,96$ & Médio & 0,190 \\
\hline & Não & 4 & $2,95 \pm 1,26$ & Médio & \\
\hline \multirow{2}{*}{ Engagement } & Sim & 31 & $3,92 \pm 0,85$ & Médio & 0,015 \\
\hline & Não & 4 & $2,7 \pm 1,23$ & Médio & \\
\hline
\end{tabular}

vel médio foi observado em $47,22 \%$ ( $\mathrm{N}=17$ ) dos profissionais, enquanto $36,11 \%(\mathrm{~N}=13)$ obtiveram nível de engagement alto.

Os índices de engagement no trabalho segundo o desejo de desistir e a satisfação com o programa de residência médica em Pediatria são apresentados na Tabela 4. Observou-se que os valores obtidos foram maiores entre os profissionais que nunca pensaram em desistir e os que se disseram satisfeitos com os programas para todas as dimensões da Uwes. Destaca-se que ambos os grupos apresentaram índices médios de engagement no trabalho para as dimensões Vigor e Absorção. Nas dimensões Dedicação e Engagement, os índices foram médios para os que já pensaram em desistir do programa e altos para aqueles que nunca pensaram em desistir.

Sobre a satisfação com o programa, os resultados foram: na dimensão Dedicação, índice alto para os satisfeitos e médio para os não satisfeitos (4,49 e 2,6, respectivamente); nas dimensões Vigor, Absorção e Engagement, os índices foram médios para ambos os grupos: 3,66, 3,68 e 3,92, respectivamente, para os satisfeitos, e 2,95, 2,54 e 2,7, respectivamente, para os não satisfeitos.

\section{DISCUSSÃO}

O engagement no trabalho é considerado um conceito recente, principalmente em relação à realidade brasileira. É importante, portanto, delimitar com mais precisão os fatores individuais, sociais, organizacionais e laborais que interferem tanto positiva quanto negativamente no desenvolvimento do constructo, em especial no que se refere à atividade médica ${ }^{13}$, cujo exercício profissional se dá em ambientes formados por intensos estímulos emocionais e físicos que acompanham o adoecer, como: contato frequente com a dor e o sofrimento, lidar com a intimidade corporal e emocional, atendimento de pacientes graves, lidar com pacientes rebeldes e não aderentes ao tratamento, hostis, reivindicadores, autodestrutivos e/ou cronicamente deprimidos, e lidar com as incertezas e limitações do conhecimento médico e do sistema assistencial, que se contrapõem às demandas e expectativas dos pacientes e familiares, que desejam certezas e garantias ${ }^{1-3,5-6}$.

Em relação à formação médica em serviço, trata-se de um período de capacitação profissional que exige que o residente faça um balanço entre o desejo de cuidar, o desejo de curar e o lidar com sentimentos de desamparo em relação ao complexo sistema assistencial, estabelecendo os limites de sua identidade pessoal e profissional ${ }^{2,5}$.

Para Pantaleoni et al. ${ }^{14}$, a transição para um ambiente com altas demandas físicas, intelectuais e emocionais gera desgaste profissional e contribui para o aumento da prevalência de problemas como burnout, que permanecem altos durante todo o período da residência. 
Apesar de todas essas dificuldades inerentes ao processo de trabalho do residente médico, os resultados deste estudo mostraram grande satisfação dos profissionais com o programa, apontada por $88,89 \%$ dos residentes, embora o desejo de desistir do programa, em algum momento, tenha sido referido por $52,78 \%$ dos profissionais.

Um estudo sobre fatores de influência na satisfação com a vida e o trabalho entre cirurgiões identificou que os profissionais apresentavam níveis moderados de satisfação no trabalho e qualidade de vida. Para os autores, o envolvimento dos profissionais no trabalho contribui para criar um ambiente mais favorável e melhorar o desempenho profissional. Portanto, é importante que gestores e supervisores institucionais invistam em ações que aumentem o engagement no trabalho ${ }^{15}$.

Alguns autores apontam que não há, nas organizações, um conjunto de benefícios preestabelecidos, recursos ou técnicas que funcionem de forma igual em distintos contextos. Destacam, ainda, que as boas práticas para o desenvolvimento de recursos humanos em organizações saudáveis são específicas para cada contexto ${ }^{16,17}$. De forma geral, a promoção da saúde laboral tanto evita os aspectos negativos do contexto ocupacional, como promove os positivos. Sendo assim, as organizações deveriam entender a saúde como um valor estratégico, pois incrementar os recursos laborais previne riscos psicossociais e amplifica a qualidade do trabalho ${ }^{10,17-19}$, mesmo porque há um desafio permanente aos trabalhadores para se adaptarem às novas contingências de trabalho e se manterem saudáveis ${ }^{13}$

Os resultados do Coeficiente Alfa de Cronbach apontam que a Uwes e suas dimensões apresentaram consistência interna adequada, em consonância com os resultados de outros estudos $^{7,18-20}$.

Os residentes estudados apresentaram níveis médios de engagement no trabalho para as dimensões Vigor, Absorção e Engagement. Estes resultados, contudo, são inferiores aos encontrados em outros estudos com profissionais de saúde colombianos ${ }^{21}$ e com profissionais da atenção primária à saúde de Portugal $^{22}$. Embora na dimensão Dedicação os resultados tenham sido ligeiramente mais altos, os valores deste estudo também são inferiores aos encontrados em outros estudos com populações semelhantes ${ }^{21-22}$.

O engagement no trabalho está relacionado com a felicidade e o bem-estar no trabalho e possui forte associação com a segurança e mortalidade de pacientes ${ }^{23}$ e com bons resultados

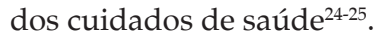

Recursos como apoio social, autonomia e envolvimento na tomada de decisões podem ser benéficos, ajudando os profissionaisa lidar com o estresse e os problemas inerentes ao ambiente e ao processo de trabalho, contribuindo para o aumento do engagement no trabalho ${ }^{22}$.

Corroborando os resultados de Ortiz e Jaramillo ${ }^{21}$, este estudo revela a importância de avaliar o comportamento profissional em uma perspectiva positiva, contribuindo para a elaboração e disseminação de ações que promovam o desenvolvimento integral dos profissionais a partir das boas experiências dos programas de formação em serviço. Para os autores, a avaliação do engagement no trabalho permite evidenciar aspectos psicossociais importantes da relação dos profissionais com o processo de trabalho, podendo apresentar um impacto positivo no âmbito organizacional, além de permitir a implementação de medidas de proteção à saúde dos trabalhadores ${ }^{21}$.

Se, por um lado, muitas vezes, os residentes enfrentam más condições de trabalho e apresentam dificuldades de enfrentamento do estresse ${ }^{2,5}$, que afeta negativamente a relação médico-paciente e o desempenho profissional, por outro, os resultados deste estudo apontam bom envolvimento dos profissionais com o ambiente laboral e, consequentemente, com o processo de formação, em especial no domínio Dedicação, que compreende níveis altos de energia e alta capacidade para lidar com os problemas (resiliência), desejo de esforçar-se sem fadigar-se e capacidade de persistir diante das dificuldades ${ }^{9,13}$.

Os maiores índices de engagement no trabalho encontrados entre profissionais satisfeitos com a residência e que nunca pensaram em desistir do programa corroboram os resultados de um estudo sobre qualidade de vida de médicos residentes, que mostrou que os profissionais apresentavam bons níveis de qualidade de vida, satisfação com a vida, bom nível de independência e boa estrutura espiritual/religiosa ${ }^{26}$.

Aspectos positivos e negativos mostrados por estudos com residentes médicos ${ }^{1,2,5-6,14,26}$ evidenciam que o suporte organizacional, de colegas e da chefia pode influenciar emoções vivenciadas no ambiente laboral econtribuir para que o trabalhador desenvolva seus potenciais e tenha experiências positivas, de realização ${ }^{27}$.

Considerando que a residência médica é um processo desgastante, com altos níveis de estresse profissional que podem causar problemas de saúde e interferir na qualidade de vida dos profissionais ${ }^{2,5}$ e na qualidade da assistência prestada aos usuários, fica evidente a relevância de novos estudos sobre engagement no trabalho de residentes médicos, colaborando com a construção de uma visão mais positiva sobre a atuação destes profissionais e o seu envolvimento com o trabalho, no contexto da formação em serviço. Assim, a preocupação com a formação médica ganharia força ao ser direcionada para a saúde dos residentes e não somente para a doença, atendendo ao 
proposto por alguns autores que destacam a importância de explorar os pontos fortes e as condições de bem-estar e envolvimento, satisfação e emoções positivas dos profissionais ${ }^{9,13}$.

Embora seja um constructo novo, o engagement se apresenta favorável aos interesses organizacionais e ao crescimento profissional dos trabalhadores. Portanto, novos estudos sobre fatores associados ao engagement no trabalho de residentes médicos poderão trazer informações sobre a relação entre estes profissionais e o processo de trabalho em diferentes contextos organizacionais, contribuindo para a compreensão do impacto das diferenças culturais e organizacionais, experiências e vícios, e tipos de intervenções que podem ajudar a desenvolver o engagement destes profissionais, aprimorando o processo de formação em serviço proposto na residência médica.

\section{CONCLUSÃO}

Os residentes deste estudo apresentaram bons índices de engagement no trabalho, principalmente no domínio Dedicação, que alcançou valores médios e altos. Há um percentual pequeno de profissionais com baixo índice de engagement em todos os domínios da Uwes.

Considerando que o engagement no trabalho é um constructo recente e a importância do processo de formação dos profissionais médicos durante a residência, novos estudos poderão contribuirpara aprimorar o conhecimento sobre o envolvimento destes profissionais com o ambiente laboral, estimulando o desenvolvimento dos aspectos positivos e interferindo nos negativos, como os desgastes que podem ser causados pela alta complexidade dos atendimentos inerentes a um hospital de ensino de alta complexidade.

Além disso, novos estudos poderão contribuir para o aprimoramento dos programas de residência médica em Pediatria e a melhoria do perfil dos profissionais inseridos no mercado de trabalho.

\section{REFERÊNCIAS}

1. Velho MTAC, Haeffner LB, Santos FG, Silva LC, Weinmann ARM. Residência médica em um hospital universitário: a visão dos residentes. Rev Bras Educ Médica. 2012;36(3):351-7.

2. Lourenção LG, Moscardini AC, Soler ZASG. Health and quality of life of medical residents. Rev Assoc Med Bras. 2010;56(1):81-90.

3. Ribeiro MA. Apontamentos sobre residência médica no Brasil. Brasília (DF): Câmara dos Deputados - Consultoria Legislativa, 2011. Disponível em: http://www2.camara.leg.br/documentos-e-pesquisa/publicacoes/estnottec/areas-da-conle/tema11/2011_123.pdf. Acesso em 16 jun. 2016.
4. Michel JL, Junior AL, Santos RA, Oliveira RA, Rebelatto JR, Nunes MP. Residência médica no Brasil: panorama geral das especialidades e áreas de atuação reconhecidas, situação de financiamento público e de vagas oferecidas. Cadernos Abem. 2011;7:13-27.

5. Nogueira-Martins LA. Qualidade de vida de médicos residentes: revisão de estudos brasileiros. Cadernos ABEM. 2010;6:12-8.

6. Dias BA, Pereira MN, Sousa IF, Almeida RJ. Qualidade de vida de médicos residentes de um hospital escola. Sci Med. 2016;26(1):ID22315

7. Rodríguez-Montalbán R, Martínez-Lugo M, Sánchez-Cardona I. Análisis de las propiedades psicométricas de la Utrecht Work Engagement Scale en una muestra de trabajadores en Puerto Rico. Univ. Psychol. 2014;13(4):1255-66.

8. Machado PGB, Porto-Martins PC, Amorim C. Engagement no trabalho entre profissionais da educação. Rev. Intersaberes. 2012;7(13):193-214.

9. Salanova M, Schaufeli WB. El engagement en el trabajo. Madrid: Alianza; 2009.

10. Bakker AB, Albrecht SL, Leiter MP. Key questions regarding work engagement. Eur J Work Organ Psy. 2011;20(1):4-28.

11. Porto-Martins PC, Basso-Machado PG, Benevides-Pereira AMT. Engagement no trabalho: uma discussão teórica. Fractal, Rev. Psicol. 2013;25(3): 629-44.

12. Agnst R, Benevides-Pereira AMT, Porto-Martins PC. Utrecht Work Engagement Scale. Tradução de W. B. Schaufeli e A. B. Bakker. Curitiba: GEPEB, 2009.

13. Porto-Martins PC, Basso-Machado PG. Engagement em el trabajo. Estud. pesq. psicol. 2010;10(3):972-6.

14. Pantaleoni JL, Augustine EM, Soukers BM, Bachrach LK. Burnout in Pediatric Residents Over a 2-Year Period: A Longitudinal Study. Acad Pediatr. 2014;14(2):167-72.

15. Mache S, Vitzthum K, Klapp B, Danzer G. Surgeons' work engagement: Influencing factors and relations to job and life satisfaction. Surgeon. 2014;12(4):181-90.

16. Lisbona A, Morales J, Palací F. El engagement como resultado de la socialización organizacional. Rev Int Psicol Ter Psicol. 2009;9(1):89-100.

17. Salanova M, Schaufeli WB. El engagement en el trabajo. Madrid: Alianza Editorial, 2009.

18. Christian MS, Garza AS, Slaughter JE. Work engagement: A quantitative review and test of its relations with task and contextual performance. Pers J. 2011;64(1):89-136.

19. Halbesleben JR. A meta-analysis of work engagement: relationships with burnout, demands, resources, and consequences. Work engagement: A handbook of essential theory and research. 2010;8:102-17. 
20. Gilchrist RM, Villalobos CEP, Fernandez LR. Estructura factorial y consistencia interna de la Utrech Work Engagement Scale (UWES) 17 entre trabajadores sanitarios de Chile. Liber. 2013;19(2):163-71.

21. Ortiz FA, Jaramillo VA. Factores de riesgo psicosocial y compromiso (engagement) con el trabajo en una organización del sector salud de la ciudad de Cali, Colombia. Acta colomb psicol. 2012;16(1):43-56.

22. Garcia SAP. Engagement no trabalho dos profissionais das Unidades de Cuidados de Saúde Primários e a sua relação com a Performance e a Satisfação no trabalho. Lisboa; 2012. Mestrado [Dissertação] — Instituto Universitário de Lisboa.

23. Bargagliotti AL. Work engagement in nursing: a concept analysis. Journal of Advanced Nursing 2012;68(6):1414-28.

24. Simpson MR. Development and psychometric evaluation of the Core Nurse Resource Scale. Journal of Nursing Management 2010;18(8):1048-59.

25. Simpson MR. Engagement at work: a review of the literature. Int J Nurs Stud. 2009; 46(7):1012-24.

26. Lourenção LG. Qualidade de vida de médicos residentes, aprimorandos e aperfeiçoandos da Faculdade de Medicina de São José do Rio Preto/SP. São José do Rio Preto; 2009. Doutorado [Tese] - Faculdade de Medicina de São José do Rio Preto.

27. Santos GB, Ceballos AGC. Bem-estar e trabalho: estudo de revisão. Psicol. estud. 2013;18(2):247-55.

\section{CONFLITO DE INTERESSES}

Os autores declaram não haver conflito de interesses.

\section{CONTRIBUIÇÃO DOS AUTORES}

Luciano Garcia Lourenção contribuiu para a concepção do projeto, análise e interpretação dos dados e redação do artigo. Cláudia Eli Gazetta, Maria Helena Pinto e Denise Beretta contribuíram para a redação do artigo, revisão crítica relevante do conteúdo intelectual e aprovação final da versão a ser publicada. Priscila Regina Teixeira, Elizangela Gianini Gonzales e Lilian Peres da Silva contribuíram com a coleta, análise e interpretação dos dados, e com a revisão crítica relevante do conteúdo intelectual.

\section{ENDEREÇO PARA CORRESPONDÊNCIA}

Luciano Garcia Lourenção

Rua Barão do Rio Branco, 745

Centro - Tanabi

CEP 15170-000 - SP

E-mail: luciano.famerp@gmail.com 